\title{
Developing Aysoo: a mobile-based self-management application for people living with HIV
}

Reza Safdari ${ }^{1}$, SeyedAhmad SeyedAlinaghi ${ }^{2}$, Niloofar Mohammadzadeh ${ }^{1}$, Tayebeh Noori ${ }^{3}$, Pakzad Rahmati ${ }^{4}$, Kowsar Qaderi ${ }^{5}$, Fabricio Voltarelli ${ }^{6}$, Esmaeil Mehraeen ${ }^{7}$

${ }^{1}$ Department of Health Information Management, Tehran University of Medical Sciences, Tehran, Iran

${ }^{2}$ Iranian Research Center for HIV/AIDS, Iranian Institute for Reduction of High-Risk Behaviors, Tehran University of Medical Sciences, Tehran, Iran

${ }^{3}$ School of Allied Medical Sciences, Zabol University of Medical Sciences, Zabol, Iran

${ }^{4}$ Iran University of Medical Sciences, Tehran, Iran

${ }^{5}$ Kermanshah University of Medical Sciences, Kermanshah, Iran

${ }^{6}$ Graduation Program of Health Sciences, Faculty of Medicine, Federal University of Mato Grosso, Cuiabá, Brazil

${ }^{7}$ Department of Health Information Technology, Khalkhal University of Medical Sciences, Khalkhal, Iran

\begin{abstract}
Introduction: A mobile-based application is one of the potential solutions to manage conditions of people living with human immunodeficiency virus (HIV) in real-time. This study aimed to design and develop HIV self-management mobile application as a suitable solution for HIV self-management of people living with HIV.

Material and methods: This study was a mixed-method in nature, carried out in five phases: 1) comparative study of existing mobile applications; 2) developed its' object-oriented conceptual model; 3) developed the initial version approved for production; 4) implementation of the final version of Aysoo, and 5) evaluation. Statistical population consisted of HIV-positive people who attended the Iranian Research Center for HIV/AIDS (IRCHA) in 2019.

Results: Aysoo was developed for appropriate HIV self-management, which provided the following main functionalities: registration of clinical symptoms, HIV/acquired immune deficiency syndrome (AIDS) management information, educational information in different stages of HIV, specific care plans based on HIV viral load, reminders to encourage medication adherence, physical activities, nutrition, and in-person visits.

Conclusions: HIV mobile-based self-management application Aysoo, has been developed in compliance with health information management (HIM) and infectious diseases experts' opinions. Aysoo is expected to be helpful in managing HIV condition and increase medication adherence of people living with HIV.
\end{abstract}

HIV AIDS Rev 2022; 21, $1: 24-30$

DOI: https://doi.org/10.5114/hivar.2022.113389

Key words: HIV/AIDS, mobile application, self-management, education, development.

Address for correspondence: Dr. Esmaeil Mehraeen, Department of Health Information Technology,

Khalkhal University of Medical Sciences, Khalkhal, Iran,

e-mail: es.mehraeen@gmail.com
Article history:

Received: 25.11 .2020

Received in revised form: 09.03.2021

Accepted: 10.03.2021

Available online: 30.11 .2021
International Journal of HIV-Related Problems

HIV \& AIDS

R e v i e w 


\section{Introduction}

According to the World Health Organization (WHO), in recent years, about 36.7 million people are living with human immunodeficiency virus (HIV), including 1.8 million children, which indicates HIV as one of the most important health concerns [1]. Since the start of HIV epidemic, about 78 million people have been infected with the virus, and 35 million have died from acquired immune deficiency syndrome (AIDS)-related diseases [2]. Therefore, healthcare organizations must undertake effective operational strategies $[3,4]$ to prevent the progression of HIV infection and reduce the number of people living with HIV (PLHIV) [5]. Self-management is one of the new operational strategies for HIV infection management that can include items, such as control of clinical symptoms, adherence to antiretroviral therapy, and educational information for PLHIV $[6,7]$.

HIV self-management strategies refer to aspects, including knowledge of disease conditions, increase of motivation, and self-awareness and self-efficacy improvement [8]. As shown in previous studies, the more awareness about the disease, the more self-management and higher selfefficacy. Based on similar studies, the use of self-management strategies have led to an increase in PLHIV motivation to continue treatment, being more engaged in providing healthcare, and improve their own health condition [9-11].

In recent years, the development of mobile-based applications has led to the use of these technologies in various areas of healthcare industry [10]. Evidence is growing supporting the effectiveness of mobile-based applications for chronic diseases management, including HIV, type II diabetes, cardiovascular diseases, and obesity [12-14]. For example, mixed-method studies found that mobile-based applications for HIV care led to statistically significant improvements in medication regimen adherence and facilitated communication with healthcare providers $[5,15,16]$. Mobile-based applications provided strategies to better manage symptoms of the disease and improve overall health-related quality of life. However, considering the limited use of mobile-based applications for chronic disease self-management and lack of mobile-based self-management application for HIV in Iran $[5,17,18]$, the present study was aimed to design and develop a mobile-based self-management application (Aysoo) for PLHIV.

\section{Material and methods}

The present study was a mixed-method research to design and develop a mobile-based self-management application for PLHIV. The study was conducted in five phases.

\section{Phase 1}

Comparative study: In the first phase of the present study, we searched for related articles and identified characteristics of HIV mobile-based self-management applications. In this stage, we analyzed features of 25 applications due to easy installation and availability (HIV/AIDS Info, AIDSinfo HIV/AIDS Guidelines, HIV clinical guide, HIV/AIDS Test, Liverpool HIV iChart, Nigeria HIV Guideline, HIVHCV Drug Therapy Guide, NACO AIDS APP, HIV-AIDS Test App, HIV-TEST, HIV Support, Anti-HIV Med, WHO HIV Tx, HIV AIDS Care, HIV Sathi, HIV Dating, Stop HIV, Tanzania HIV Guideline, Avoid HIV and AIDS, How to cure HIV, HIV/AIDS Pakistan, HIV/AIDS info Somali, HIV NEED TO KNOW, HIV Management in Australia, and HIV HELP). We extracted all their features and attributes, registered on a data extracted form, and classified them as common functions of HIV mobile-based application.

These common extracted features were selected for an initial model of Aysoo. To assess validity of the selected features and attributes, a research-made questionnaire was developed. The questionnaire consisted of 37 questions that were based on a 5-point Likert scale, with 'completely agree', 'agree,', 'no idea, 'disagree', and 'completely disagree'. Validity of content of the questionnaire was measured by four experts in health information management and five infectious diseases specialists. To ensure reliability of the questionnaire, it was completed by nine of the above-mentioned specialists and experts; they were requested to complete the questionnaire for again after 10 days. Collected data were analyzed with SPSS, and reliability of the questionnaire was calculated as 0.89 using Cronbach's $\alpha$. Six experts of health information management (HIM) and 15 infectious diseases specialists completed the questionnaire and then, obtained results were analyzed. In the final step of this phase, comparative modal of Aryan was prepared for study participants.

\section{Phase 2}

Developing object-oriented model of Aysoo: In the first step of this phase, to design conceptual model of initial version of Aysoo, statistical population decided to select common features of comparative model (with $95 \%$ of minimum agreement). Consequently, 35 features were selected. In the second step, unified modeling language (UML) diagrams (workflow, class, and activity diagrams) were used to design conceptual model of Aysoo.

\section{Phase 3}

Developing the initial version: In this phase, the initial version of HIV mobile-based self-management application was developed according to findings of comparative and conceptual model. The developed application can be installed on Android operating system version 4-7.

\section{Phase 4}

Implementation: During this phase, the original developed version of mobile-based self-management application was implemented at the Faculty of Paramedical Sciences and the Iranian Research Center for HIV/AIDS (IRCHA). 


\section{Phase 5}

Evaluation: A usability evaluation was conducted to determine user-friendliness, ease of use, level of security, and motivational impact of usability of Aysoo application from PLHIV perspective. For this purpose, software usability measurement inventory (SUMI) as a standard questionnaire for assessing quality of use of application by end users was applied [19]. In this phase, statistical population included all PLHIV who attended the IRCHA in $2019(n=65)$, that had a smartphone, and entered the study with an informed consent. The self-management application was provided to the study participants who were requested to use it for 30 days. After this period, dimensions of usability were evaluated from PLHIV perspectives. Collected data were analyzed using SPSS version 19 (SPSS Inc., Chicago, Illinois, USA) and descriptive statistics (frequency and mean).

\section{Results}

By using a content analysis of 25 different applications, 37 features were extracted. Some of them had frequently been used in various mobile applications, but there were some features, which had been used exclusively in some models and received special data from its' user. Based on these findings, the required features of the initial version design of Aysoo application were considered as follow: registration of clinical symptoms, ability to provide specific care plan for each individual user, providing HIV/ AIDS management information, antiretroviral therapy suggestions (specific care plans based on the number of HIV per microliter of blood), reminders to encourage medication adherence, educational information in different stages of HIV, physical activities and nutrition, and reminders for in-person visits, periodical tests, and vaccinations. Workflow diagram of Aysoo is shown in Figure 1.

The instruction on how to perform tasks in Aysoo application were as follow:

- Input

Inputs are directly entered by a user; including username, password, demographic, and clinical data (age, sex, weight, fever), physiologic information, diet, and test results.

\section{- Process}

Patient's data processed through formulas, such as calculating body mass index (BMI), medication dose, daily intake of calories, daily distribution of calories, and HIV viral load (calculation number of HIV per microliter of blood).

- Output

Output shows the results of data analysis and reports:

- diagrams: number of HIV and BMI;

- various calculation reports: changes in weight and BMI, and number of HIV;

- reminders: medication administration, physical activities, in-person visits, periodical tests, and vaccinations;

- calculation results: BMI, required physical activity, daily diet regimen, and disease stage (Figure 2).

A sample of user-interface screens of HIV mobile-based self-management application Aysoo is presented in Figure 3. As shown in Figure 4, the HIV mobile-based self-management application was considered as user-friendly in moderate level from viewpoints of most users $(n=55,84.6 \%)$. Moreover, most users $(n=53,81.5 \%)$ evaluated motivational and educational effects of Aysoo application as 'good'.

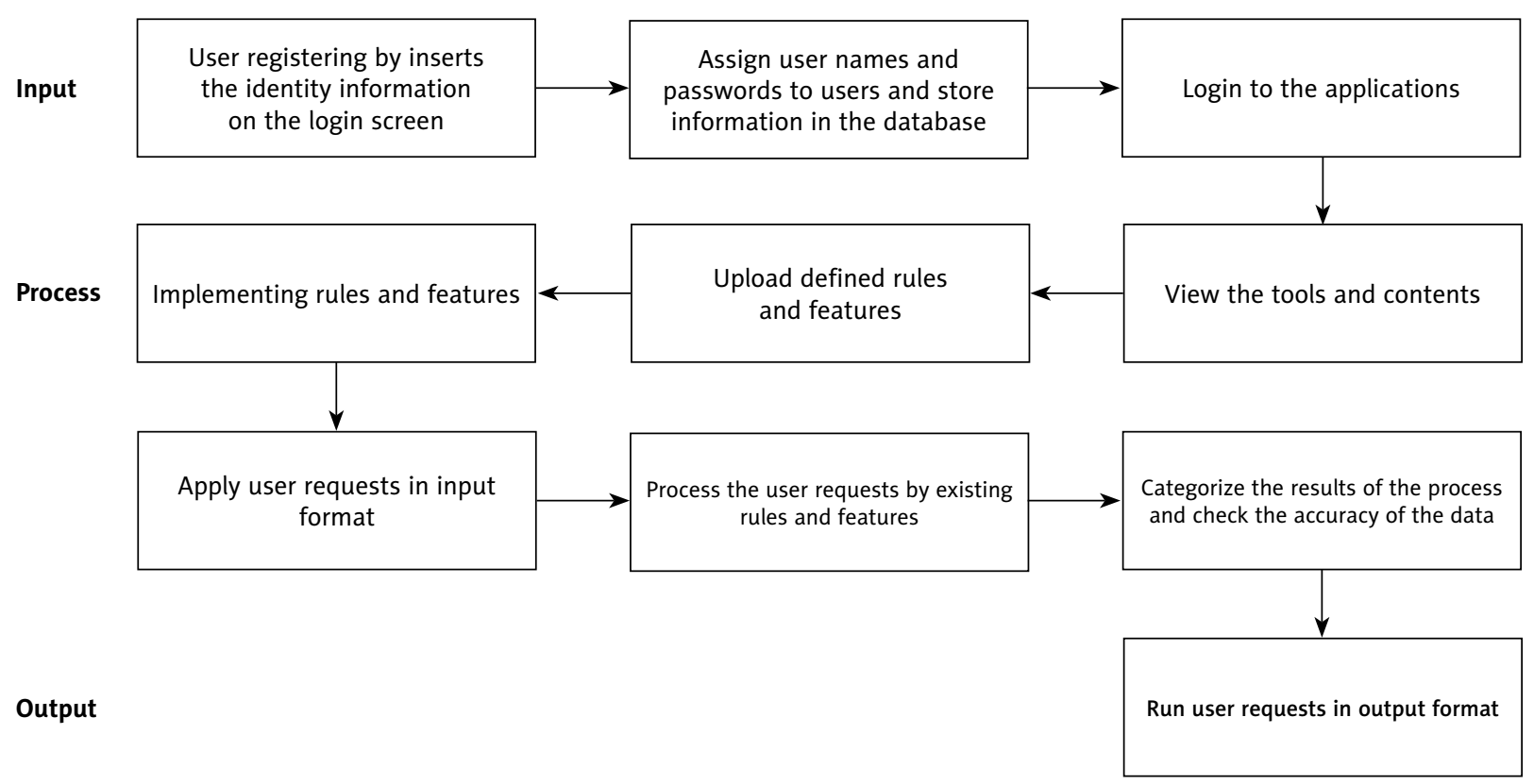

Figure 1. Workflow diagram of HIV mobile-based self-management application Aysoo 


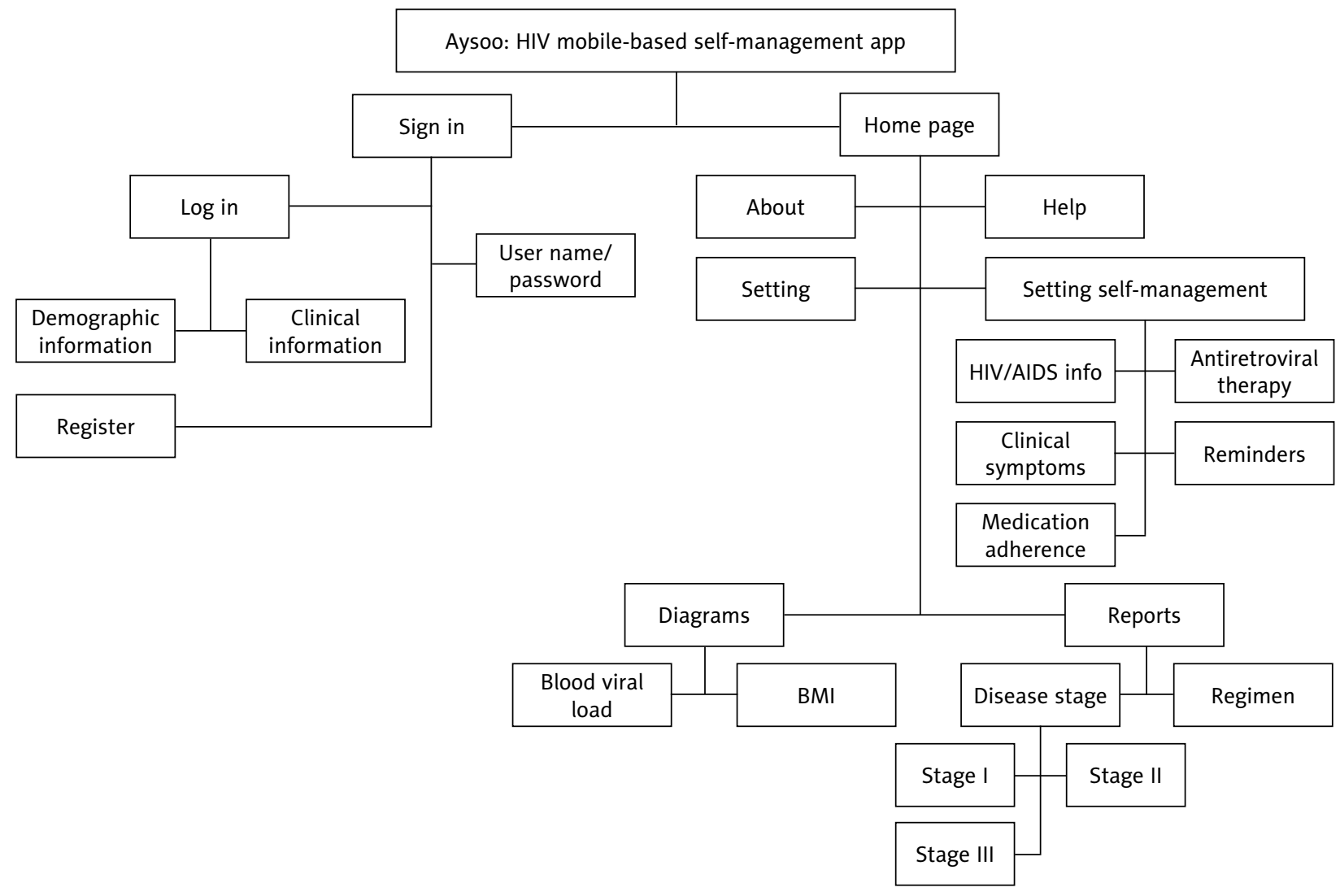

Figure 2. Model of HIV mobile-based self-management application Aysoo
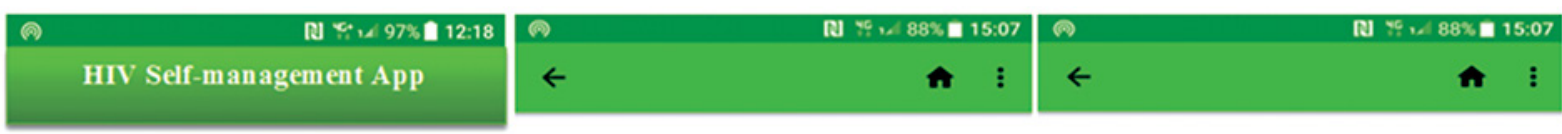

\section{Sign in}
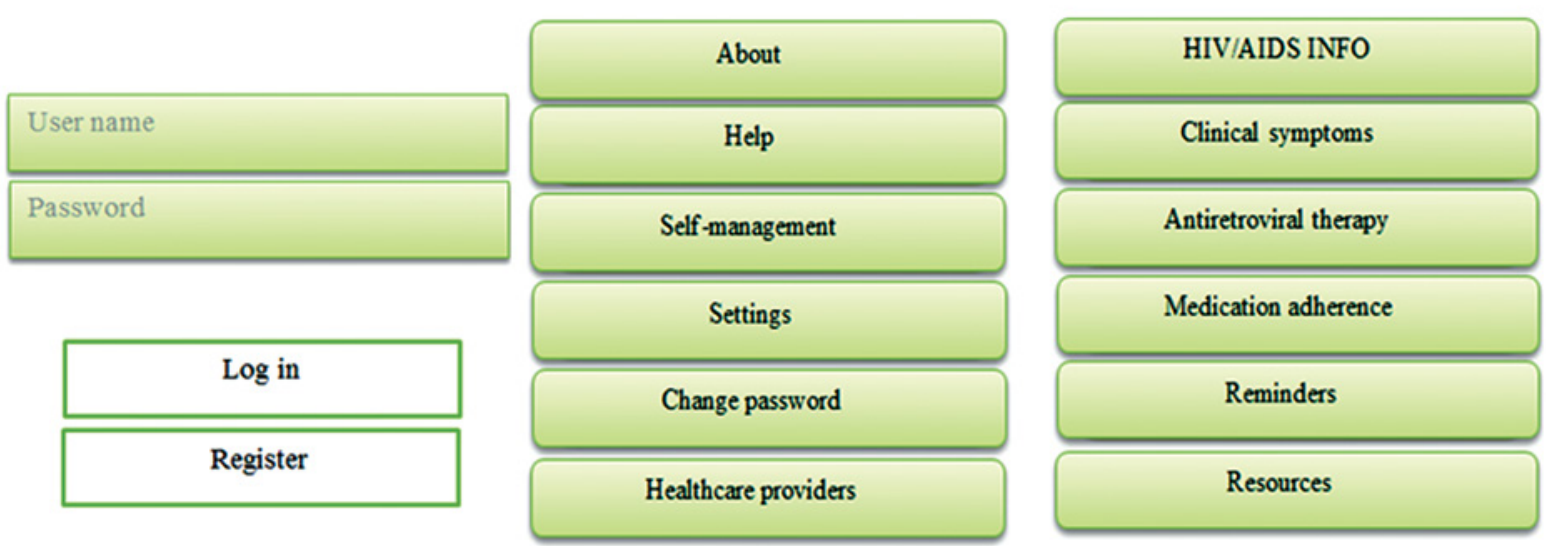

Figure 3. User interface screen of Aysoo

\section{Discussion}

The eagerness of healthcare providers in using mobile technology and HIV self-management is remarkable. Con- sequently, Aysoo functions allows for managing medication adherence, physical activity, diet, periodical tests, vaccinations, and in-person visits. However, the prospective of technology-based applications to provide healthcare facilities and 


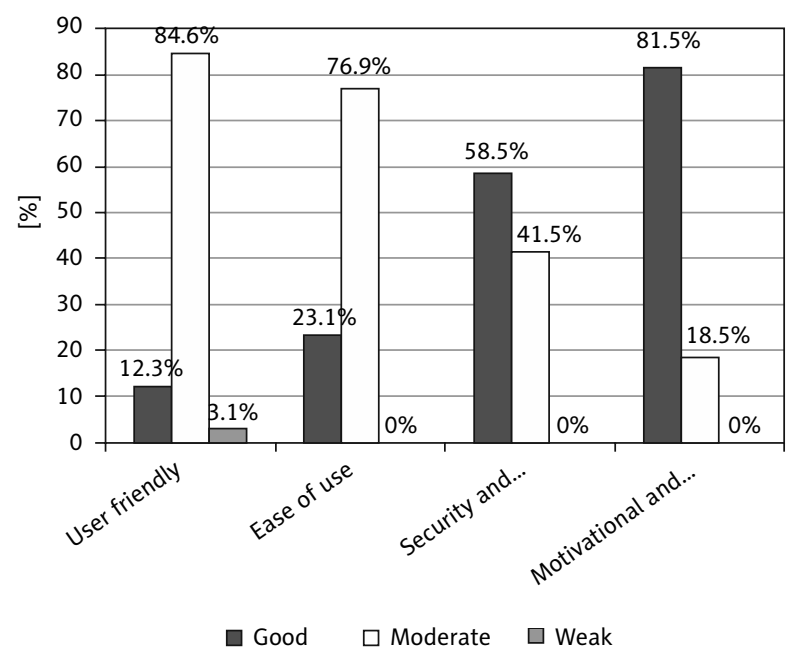

Figure 4. Aysoo usability percentage from the users' perspectives

enhance self-management interventions have been well-documented over the recent decade. Farzandipour et al. in their systematic review reported multifunctional mobile-based applications presenting good potential in the management of chronic conditions and in improving quality of life of affected patients compared with traditional solutions [20]. In a study by Garofalo et al., it was indicated that the use of mobile-based applications for self-management of people living with HIV can ensure care tracking, prevention/interventions programs, medication, and clinical check-ups [21]. Focus group findings from a study by Ramanathan et al. with HIV-positive individuals and young mothers, demonstrated that mobile-based applications can provide social and psychological facilities for HIV-positive persons and improve their quality of healthcare received [22].

In Aysoo, user chooses applicable features, such as registration of clinical symptoms, receiving educational information, antiretroviral therapy suggestions, reminders to encourage medication adherence, in-person visits, periodical tests, and vaccinations. Swendeman $e t$ al. findings also showed that functional properties of mobile-based self-management application with medication and dietary reminders could improve self-care and self-awareness skills of PLHIV by increasing the understanding of existing conditions of these people and enhance their motivation [23]. To develop an effective mobile-based application, it is essential to identify users' requirements of app's design, so that they can be more engaged in using the application [24]. Schnall et al. reported that tracking the results of tests and reporting on health status were the functional properties of mobile health technology used as a tool for self-care and self-management of PLHIV. The results of this similar research also showed that mobile technology can play an important social role, providing capabilities of textual as well as audio and video communication between users, thereby facilitating the exchange of healthcare experiences [25].
One of the cornerstones in HIV management is on-time medication administration as an important factor in adherence to treatment. Mobile-based interventions improve HIV medication adherence, as they can trigger timely action. By using Aysoo's reminder feature, the user can take the prescribed medicines on time (based on disease stage and healthcare providers' instructions). Similar studies have emphasized that in developing countries, where the number of PLHIV is significant, the expansion of technology-based strategies to improve antiretroviral treatment and increase adherence to treatment is a vital self-management strategy [26, 27].

Technology-based clinical symptoms' management, with self-care strategies, was shown to be effective in PLHIV [28], and behavior change in the context of healthcare using mobile-based interventions have been well-documented. Therefore, employing strategies for HIV clinical symptoms' management is the strength of the content of mobile-based self-management application [24]. Using Aysoo, users can register their clinical symptoms, and healthcare providers can manage the appeared symptoms subsequently. A similar study by Schnall et al. reported that use of mobile-based self-management tool can improve symptoms' experience of PLHIV [29]. A 2018 related study by Latif et al. showed that mobile-based health solutions can be applied to reduce rising burden of infectious diseases, such as HIV/AIDS and tuberculosis, with clinical symptoms' management and focusing on behavioral change programs to promote healthy lifestyle [30].

The result of usability evaluation indicated that PLHIV had positive perceptions of Aysoo as mobile application, which provided user-friendly features with motivational and educational effects, and security and reliability. Westergaard et al. evaluated adoption of a mobile-based health intervention to increase coordination of healthcare services for PLHIV. The results of this research showed that usability of mobile-based health interventions was helpful for PLHIV to participate in self-management strategies [31]. A study by Swendeman et al. demonstrated that in some of participants, the evaluation of mobile-based self-care application was very useful due to availability and comfort of use. Moreover, the results of this evaluation showed that the use of smartphone-based self-care applications increased the awareness and behavior modification of PLHIV [23]. Another similar study that evaluated cell phone-based therapeutic interventions for HIV and tuberculosis in Mozambique, indicated that mobile-based self-management application facilitated communication between healthcare providers and patients, and increased patients' awareness and motivation [32-35].

\section{Limitations}

The main limitation of the present study was the unsuitable mental state of the participants of the study, who due to their illness, were not much oriented toward the use of mobile-based self-management apps. This problem was solved by explaining the benefits of using this application. 


\section{Conclusions}

HIV mobile-based self-management application Aysoo was developed in compliance with HIM and infectious diseases experts' opinions. It is expected that Aysoo would play an effective role in improving medication adherence, provide useful educational information, progress self-management skills of PLHIV, and increase their motivation to continue treatment. Considering the increasing use of a mobile-based application in healthcare industry, it is recommended to design and develop self-management application for other diseases, such as asthma, diabetes, or tuberculosis.

\section{Acknowledgements}

This study was approved by Tehran University of Medical Sciences with the ethics code: IR.TUMS.VCR.REC.1397.139 and grant no. 97-01-55-38289. We thank all the participants for their contribution to the study.

\section{Conflict of interest}

The authors declare no conflict of interest.

\section{References}

1. Razavi P, Hajifathalian K, Saeidi B, et al. Quality of life among persons with HIV/AIDS in Iran: internal reliability and validity of an international instrument and associated factors. AIDS Res Treat 2012; 2012: 849406

2. Russell S, Martin F, Zalwango F, et al. Finding meaning: HIV selfmanagement and wellbeing among people taking antiretroviral therapy in Uganda. PLoS One 2016; 11: e0147896.

3. Babazadeh T, Moradi F, Dargahi A, Zibaee N, Rezakhani Moghadam $\mathrm{H}$, Amirian T. The effect of an educational program on knowledge, attitude and behavior of mothers in relation to proper nutrition in 2-6 year old children. Journal of Mazandaran University of Medical Sciences 2015; 25: 105-109.

4. Moghaddam HR, Allahverdipour H, Matlabi H. Successful recruitment and retention strategies for women health volunteers: viewpoints of the volunteers' supervisors and relevant researchers. J Multidiscip Healthc 2018; 11: 621-634.

5. Mehraeen E, Safdari R, SeyedAlinaghi SA, Mohammadzadeh N, Mohraz M. Common elements and features of a mobile-based selfmanagement system for people living with HIV. Electron Physician 2018; 10: 6655-6662.

6. Jebraeily M, Makhdoomi K. Factors influencing the improvement of self-management behavior in hemodialysis patients. Journal of Nephropharmacology 2018; 7: 110-113.

7. Zare Z, Jebraeily M. Patients' perceptions of applying information and communication technology tools in self-care and factors affecting it. Acta Inform Med 2018; 26: 102-105.

8. CooperV, ClatworthyJ, Whetham J;EmERGEConsortium. mHealth interventions to support self-management in HIV: a systematic review. Open AIDS J 2017; 11: 119-132.

9. Khumsaen N, Stephenson R. Adaptation of the HIV/AIDS selfmanagement education program for men who have sex with men in Thailand: an application of the ADAPT-ITT framework. AIDS Educ Prev 2017; 29: 401-417.

10. Nematollahi M, Khalesi N, Moghaddasi H. A comparative study of management information system for HIV/AIDS in selected countries. Payesh 2012; 11: 425-433.
11. Webel AR, Moore SM, Hanson JE, Salata RA. The rationale, design, and initial efficacy of SystemCHANGE ${ }^{\mathrm{m}}$-HIV: a systems-based intervention to improve physical activity in people living with HIV. J AIDS Clin Res 2013; 4: 1000200.

12. Copeland T. Self-managing HIV/AIDS: cultural competence and health among women in Nairobi, Kenya. Anthropol Med 2018; 25 : 176-190.

13. Côté J, Godin G, Ramirez-Garcia P, et al. Virtual intervention to support self-management of antiretroviral therapy among people living with HIV. J Med Internet Res 2015; 17: e6.

14. Wilhide III CC, Peeples MM, Kouyaté RCA. Evidence-based mHealth chronic disease mobile app intervention design: development of a framework. JMIR Res Protoc 2016; 5: e25.

15. de Lima Salgado A, Freire AP (eds.). Heuristic evaluation of mobile usability: a mapping study. International Conference on HumanComputer Interaction. Springer; 2014.

16. Jeon JH, Kim K. Development of mobile app for self-management performance of patients with CHB. Adv Sci Technol Lett Mech Eng 2016; 129: 229-233.

17. Kuparinen L, Silvennoinen J, Isomäki H (eds.). Introducing usability heuristics for mobile map applications. Proceedings of the $26^{\text {th }}$ International Cartographic Conference, August 25 30, 2013, Dresden, Germany. International Cartographic Association; 2013.

18. L'Engle KL, Green K, Succop SM, Laar A, Wambugu S. Scaled-up mobile phone intervention for HIV care and treatment: protocol for a facility randomized controlled trial. JMIR Res Protoc 2015; 4: e11.

19. Online SUMI questionnaire 2011. Available from: http://sumi.uxp.ie/.

20. Farzandipour M, Nabovati E, Sharif R, Arani MH, Anvari S. Patient self-management of asthma using mobile health applications: a systematic review of the functionalities and effects. Appl Clin Inform 2017; 8: 1068-1081.

21. Garofalo R, Kuhns LM, Hotton A, Johnson A, Muldoon A, Rice D. A randomized controlled trial of personalized text message reminders to promote medication adherence among HIV-positive adolescents and young adults. AIDS Behav 2016; 20: 1049-1059.

22. Ramanathan N, Swendeman D, Comulada WS, Estrin D, RotheramBorus MJ. Identifying preferences for mobile health applications for self-monitoring and self-management: focus group findings from HIV-positive persons and young mothers. Int J Med Inform 2013; 82: e38-e46.

23. Swendeman D, Ramanathan N, Baetscher L, et al. Smartphone selfmonitoring to support self-management among people living with HIV: perceived benefits and theory of change from a mixed-methods, randomized pilot study. J Acquir Immune Def Syndr 2015; 69 Suppl 1: S80-91.

24. Cho H. Development and usability evaluation of an mHealth application for symptom self-management in underserved persons living with HIV. Columbia University; 2017.

25. Schnall R, Bakken S, Rojas M, Travers J, Carballo-Dieguez A. mHealth technology as a persuasive tool for treatment, care and management of persons living with HIV. AIDS Behav 2015; 19: 81-89.

26. Nhavoto JA, Grönlund $\AA$, Klein GO. Mobile health treatment support intervention for HIV and tuberculosis in Mozambique: perspectives of patients and healthcare workers. PLoS One 2017; 12: e0176051.

27. Bachman DeSilva M, Gifford AL, Keyi X, et al. Feasibility and acceptability of a real-time adherence device among HIV-positive IDU patients in China. AIDS Res Treat 2013; 2013: 957862.

28. Schnall R, Siegel K, Jia H, Olender S, Hirshfield S. Racial and socioeconomic disparities in the symptom reporting of persons living with HIV. AIDS Care 2018; 30: 774-783.

29. Schnall R, Cho H, Pichon A, Mangone A. A mobile video information provider (VIP) for dissemination of evidence from patientcentered outcomes research for improving symptom management. The Practice of Patient Centered Care: Empowering and Engaging Patients in the Digital Era 2017; 244: 80. 
30. Latif S, Khan MY, Qayyum A, et al. Mobile technologies for managing non-communicable diseases in developing countries. Mobile Applications and Solutions for Social Inclusion: IGI Global; 2018. p. 261-87.

31. Westergaard RP, Genz A, Panico K, et al. Acceptability of a mobile health intervention to enhance HIV care coordination for patients with substance use disorders. Addict Sci Clin Pract 2017; 12: 11.

32. Nhavoto JA, Grönlund Å, Klein GO. Mobile health treatment support intervention for HIV and tuberculosis in Mozambique: perspectives of patients and healthcare workers. PLoS One 2017; 12 e0176051.

33. Mehraeen E, Safdari R, Seyedalinaghi SA, Mohammadzadeh N, Arji G. Identifying and validating requirements of a mobile-based self-management system for people living with HIV. Stud Health Technol Inform 2018; 248: 140-147.

34. Mehraeen E, Safdari R, Mohammadzadeh N, Seyedalinaghi SA, Forootan S, Mohraz M. Mobile-based applications and functionalities for self-management of people living with HIV. Stud Health Technol Inform 2018; 248: 172-179.

35. Niakan S, Mehraeen E, Noori T, Gozali E. Web and mobile based HIV prevention and intervention programs pros and cons - a review. Stud Health Technol Inform 2017; 236: 319-327. 